\title{
Spatial Delimitation, Forecasting and Control of Japanese Encephalitis: India - A Case Study
}

\author{
Shanmugavelu Sabesan*, Hari Kishan Raju Konuganti* and Vanamail Perumal
}

Vector Control Research Centre, Medical Complex, Indira Nagar, Puducherry - 605 006, India

\begin{abstract}
Japanese encephalitis (JE) is the leading cause of viral encephalitis through large parts of Asia with temperate and subtropical or tropical climate. In the present communication environmental determinants that influence the occurrence of JE have been enlisted, and based on which a conceptual frame for JE transmission was developed. The concept of endemic and epidemic has been defined using cluster analysis on JE occurrences in 175 districts over a period of 53 years in India. The average number ( \pm standard deviation) of occurrences in endemic (7.4 \pm 3.5$)$ and epidemic districts (3.4 \pm 2.9$)$ was statistically significant ( $\mathrm{t}$ ' $=8.3 ; \mathrm{P}=0.000$ ). In the epidemic areas, JE immunization of target population in the risk area may be an effective preventive measure. In the endemic areas regular monitoring of vector population and viral activity, and implementing appropriate integrated methods of vector control are likely to reduce the transmission, besides the selective immunization of children.
\end{abstract}

Keywords: Japanese encephalitis, Delimitation, Forecasting, Vector control strategy, India.

\section{INTRODUCTION}

The epidemiology of Japanese encephalitis (JE) is extremely complex, and even after 50 years of research, some aspects remain unresolved. JE is a mosquito borne viral disease known as "brain fever" with high case fatality and grave sequelae with neuropsychiatric disorders [1]. JE is the leading cause of encephalitis through large parts of Asia [2]. Primarily the disease affects children below 15 years [2]. However, adults too get infected in populations where the virus is newly introduced. There is underreporting of cases, and conservative figures estimate some 50,000 cases annually, with $25-30 \%$ mortality rate and 30\% long-term sequelae among survivors [3]. This figure is believed to represent only a small proportion of the disease burden that actually exists. Culicine mosquitoes (mainly Culex vishnui $\mathrm{gr}$.) transfer the virus to humans from infected animals, in most cases domestic pigs and wading birds. Human beings are not considered a reservoir for viral transmission [4].

There is no treatment available for the disease. An effective control or prevention of JE needs a high priority, since (a) the annual epidemics is on the increase over the past decades and the disease has spread to extensive new areas and (b) being a zoonosis, in which man is tangentially affected. Control of JE in the programme mode is carried out only as a public relation measure in many countries including India, mostly after the establishment of viraemia in the population. For any public health delivery, 'districts' are the administrative units in India. Compared to many other countries, these units are relatively larger in area $\left(\approx 5340 \mathrm{~km}^{2}\right)$ and population density (>1.7 million) [5]. And, within the district there is

*Address correspondence to these authors at the Vector Control Research Centre, Puducherry - 605 006, India; Tel: + 91413 2279072;

Mobile: +91 94432 55377; Fax: +91 413 2272041;

E-mail: sabesan1@yahoo.com

Vector Control Research Centre, Puducherry, 605 006, India;

Mobile: +91-98942 41107; E-mail: khkraju@gmail.com always sporadic occurrence of cases in relation to time and space and hence confusion exists in defining the area as 'endemic' or 'epidemic'.

In this paper, we attempt to list out the risk factors that are likely to influence the transmission and based on which a conceptual frame for JE transmission is aimed. Further, to device a sustainable and appropriate control strategy a logical approach in defining the areas as 'endemic' and 'epidemic' is made. To forecast the risk of major factor(s) that led to transmission of JE the application of space borne satellite images and geographical information system is explored.

\section{Risk Factors}

Risk factors associated with the transmission have been identified, based on the data available since 1955 till date as: (i) Change in land use pattern - from dry land crops to wet land crops (rice), (ii) Change in agriculture practices - increasing the frequency of rice cultivation, (iii) Change in socioeconomic status \& animal husbandry - promotion of pig breeding as a food source - promotion of duck farming, (iv) Park / bird sanctuaries in the region - and or bird migration involving the region, and (v) Vagaries of Climate - temperature and rainfall.

The JE incidence, however, varied in different countries, the highest occurrence is found in the rice-growing areas of the country [6]. Further, the JE mosquito vector(s) breed mainly in the rice fields, and human cases occur scattered over extended rural areas. Therefore, it is evident that there is an ecological connection with irrigation and paddy cultivation. Further, the increased water availability during a particular stage of the paddy cultivation is reported to be a premonitory sign that can lead to markedly increased population of the vector mosquitoes [6-8]. Therefore, it is evident that the physiographic factors such as land use/land cover, vegetation-type and growth, and, extent of water spread are likely to play a major role on JE transmission. Climatic factors 


\section{JE TRANSMISSION DYNAMICS}

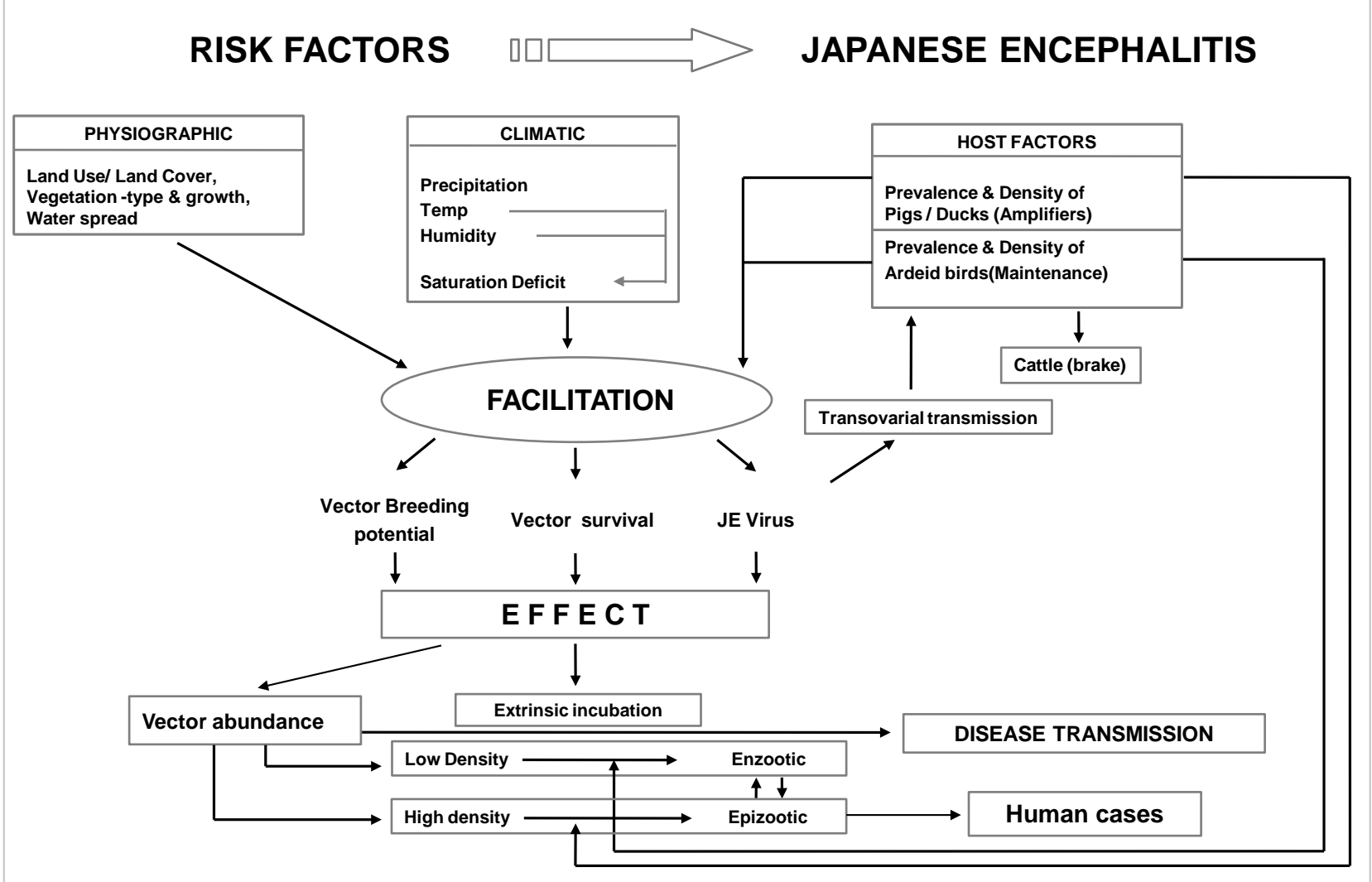

Fig. (1).

such as precipitation, temperature, and humidity are considered to be risk factors as they have direct role to facilitate mosquito population, survival and thereby their vectorial capacities [8]. Since the intensity of JE transmission depends on the amplifier-host factors (prevalence and density of pigs / ducks) and maintenance-host factors (prevalence and density of Ardeid birds), the density of these animals and the JE viraemic activity in them also form the potential risk factors for JE transmission.

\section{Conceptual Frame of JE Transmission}

A conceptual frame has been developed, incorporating all the important components of JE transmission dynamics (Fig. 1). The basic natural cycle of JE involves both vertebrate and an arthropod. JE virus is primarily maintained at low levels amongst Ardeid birds (cattle egret \& pond heron) and the cycle continues between birds and mosquito species, which feed on them. When the vector density increases enormously, a few of them spill over to humans and thereby man is an incidental and dead-end host. Man to man, transmission does not occur in nature. Cattle also act as dead-end host ('brake') in the transmission cycle. As the cattle circulate only a low level of virus, they do not develop disease. From Ardeid birds, JE is transmitted by mosquitoes to pigs / ducklings. The pigs / ducklings serve as amplifying hosts since the virus multiplies in them. Man or cattle get infected either from birds or pigs / ducklings through mosquitoes. Ardeid bird - mosquito - Ardeid bird and pig / duckling - mosquito pig / duckling cycle exist in nature. The bird cycle is more stable, persistent and enzootic. The pig cycle appears to be temporary and epizootic. The epidemic in man appears to follow pig epizootics, and this coincides especially when the vector density shoots up. Though the JE virus transmission from mosquito to mosquito via reproductive route ("transovarial' transmission) has been reported [9], it has not been considered seriously because of a very low virus titre. Still, it bears epidemiological significance, as the mosquitoes themselves maintain the virus during the non-transmission (lean) period.

\section{Pattern of Occurrence of Transmission}

JE virus activity in India was first established by a serological survey in 1952. Clinical recognition of this disease was first made in 1955 at Vellore of North Arcot district in Tamilnadu state. Between 1955 and 2007, there had been an increase and spread of infection throughout the country, except in arid and high land regions. Up to 1965 , there were sporadic incidences (52 cases) reported only from North Arcot district. Outbreaks of JE occurred in West Bengal during 1973 - 76 and in Andra Pradesh, Bihar, Tamil Nadu and Uttar Pradesh during 1977 - 79. Subsequently, outbreaks were reported in Assam, Karnataka and Pondicherry (now: Puducherry) in the early 1980s; and in Uttar Pradesh and Haryana in late 1980 s \& early 1990s. Kerala, which was free from JE till early 1990s, experienced the first outbreak in 1996 and a few cases in the following years. A new focus of infection in Sangli district of Maharashtra was reported in 1999. Since 2000, the JE occurrence has been reported from 
16 States and Union Territories and among them Uttar Pradesh, Assam and Karnataka have accounted for more than $90 \%$ of the total incidence in India [10].

The occurrence of JE in different states of India over a period of time reveals: (i) the hard core foci in the central peninsular region continue to remain for the last five decades; and (ii) the disease is spreading to newer areas in the north (except arid and high land zones). We tried to characterize the JE occurrences based on qualitative data (' 1 ' occurred and ' 0 ' not occurred) that was available from 1955 to 2007 for the entire India. The data sources were from publications of National Vector Borne Diseases Control programme, National Institute of Virology and Indian Council of Medical Research, available up to 2007. The districts in which at least one occurrence had been reported during the 53 years of observation (1955-2007) formed the data-base for the present analysis. Each yearly occurrence of JE was taken as one variable and it was in binary form ( 0 or 1$)$. Therefore, 53 variables were taken to classify the districts into two types, which are likely endemic or epidemic form. For the present analysis to classify the districts, hierarchical cluster analysis by adopting within group linkage method and squared Euclidean measure was carried out. Clustering ordering was driven by how many observations could be combined at a time or what determines that the distance is not statistically different from 0 between two observations or two clusters. The clusters were arrived at joining together similar observations (agglomerative method). The two types of districts emerged through cluster analysis were mapped (Fig. 2) and the descriptive statistics for different climatic/environmental variables were computed for their epidemiological implications.

Out of 593 districts exist in India 175 were reported to have JE occurrence at least once during the period 19552007. Among 175 districts 80 were classified as 'endemic' and the remaining 95 districts were classified as 'epidemic'. The average number ( \pm standard deviation) of occurrences in these two types was $7.4 \pm 3.5$ and $3.4 \pm 2.9$ respectively. The difference in average number of occurrences was statistically significant (Student's t-test $=8.3 ; \mathrm{P}=0.000$ ). The range in number of occurrence in endemic district was 5-18 and in epidemic districts was $1-18$. While $85 \%$ of endemic districts

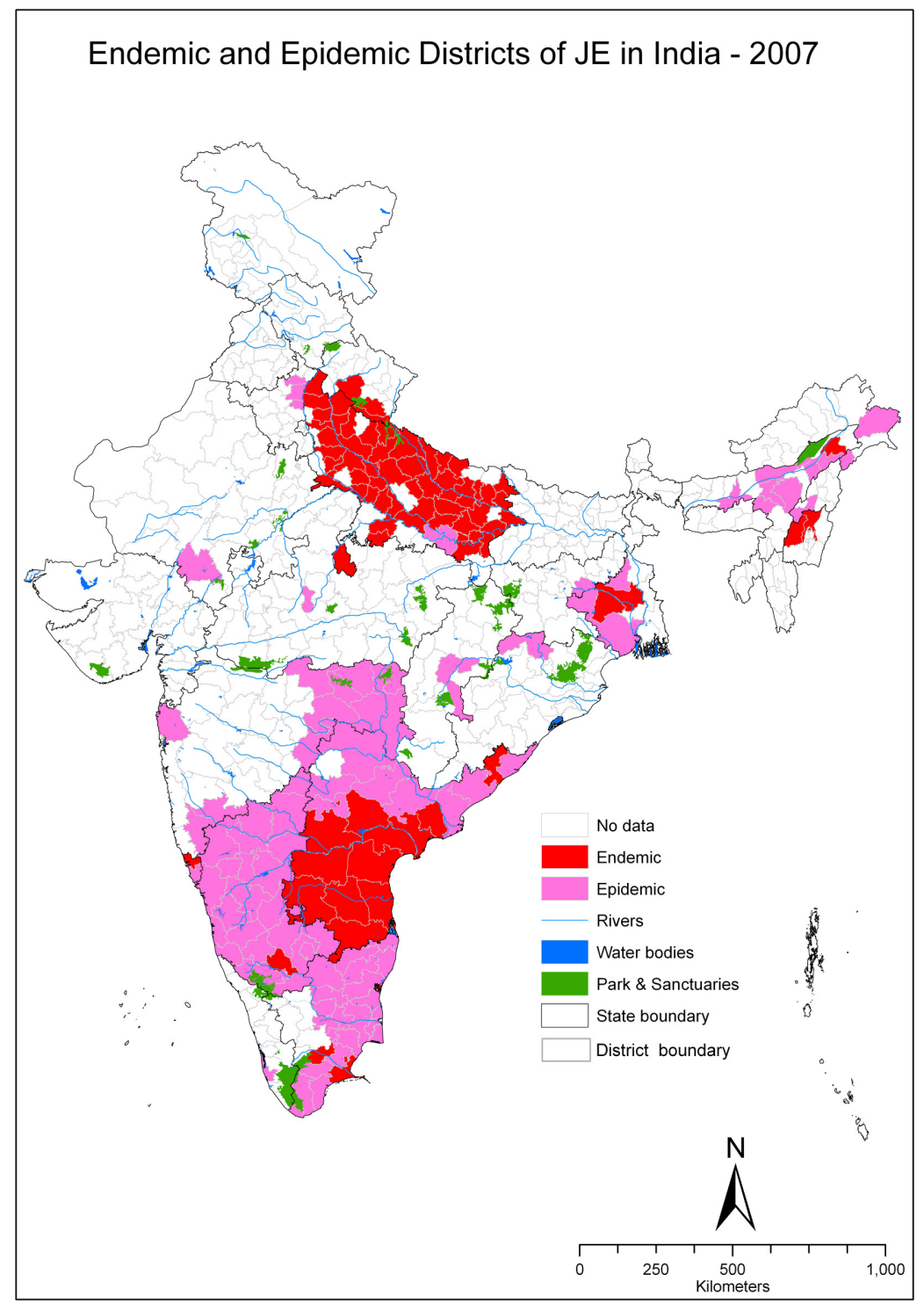

Fig. (2). 
experienced up to 11 occurrences, $87 \%$ epidemic districts experienced up to 6 occurrences only. The distribution of districts by State and the nature of occurrence of JE are shown in Fig. (2). It was observed that among 80 districts classified as endemic 54(68\%) are from Utter Pradesh state alone. Similarly, among the districts classified as epidemic, majority of them are from Karnataka (26\%) and Tamil Nadu $(22 \%)$ states. The average values of different climatic/environmental variables are presented in Table $\mathbf{1}$ for epidemic and endemic districts. It was observed that there was no significant difference between endemic and epidemic districts with respect to average temperature $(\mathrm{t}=1.43$; $\mathrm{P}=0.153)$ and relative humidity $(\mathrm{t}=1.75 ; \mathrm{P}=0.082)$. However, in the epidemic districts there was a marked increase in average rainfall and decrease in altitude compared to the endemic districts.

There is no classical definition available yet in literature in relation to space and time for JE. Therefore, an attempt has been made to define the 'endemic' and 'epidemic' form of JE in a more rational way by applying statistical technique for the empirical data. Based on the present observations, 'Endemic' JE may be defined as "Occurrence of local transmission of JE in an area repeatedly or periodically at an interval of $3-5$ years that remain at a fairly stable prevalence". Also, in endemic districts, sporadic cases occur at any point of time due to congenial climatic conditions throughout the year. 'Epidemic' JE may be defined as "Unusual occurrence of JE transmission in old foci or fresh occurrence in a newer area and generally with low incidence".

The analysis further indicates that both rainfall and altitude have a bearing on the epidemic form of JE. When the rainfall is high, the likelihood of getting JE epidemic in the low altitude districts is more. It confirms to the earlier report that the epidemics coincide with the monsoon and post monsoon period, especially when there is a heavy rainfall following severe draught conditions [11]. During this period, a large number of extensive water collections facilitate a potential ground for the breeding of mosquito vector(s) in the rice cultivation areas. Further, the late appearance of mosquito predators in the breeding habitats and the visit of wading birds to these sites promote the abundance of vectors and the viral activity (transmission) involving birds respectively.

Forecasting the Environmental Determinants of JE Transmission

'Vector abundance' is the key element for the disease outbreak, when the other transmission parameters are condu- cive. Vegetation type and growth stage of paddy, along with the extent of water availability are reported to be the important factors determining vector abundance [11]. It is impracticable to obtain the prevalence and density of the vector mosquitoes and also to monitor any sharp increase in the vector density by any manual means, as their breeding habitats are enormous. However, it would be possible to forecast the environmental determinants that influence the vector density, by using modern tools such as Remote Sensing (RS) and Geographical Information Systems (GIS). Uses of RS and GIS have already been described elsewhere for many other diseases [12-14]. These tools would provide reliable information on a desired spatial and temporal scale. The basic products that are commonly extracted from RS data include vegetation index, cold cloud duration and land surface temperature. Depending on the time of year (season), the values thus derived from the remote sensing data can be used to determine the vegetation type / growth stage of plants, the extent of water bodies and rainfall, and the condition potential for vector survival in particular sites respectively. If a spurt in vector density can be detected in advance, risk prediction will subsequently become possible, following the screening of amplifier hosts and the vector mosquito pools for virus activity.

\section{JE Control Options}

Control programmes have been focusing on three major areas: mosquito control, amplifying host (pig) control, and vaccination. However, neither mosquito control nor amplifying host (pig) control has been proven to be effective measures to control JE, as they are carried out in India mostly after the outbreaks. Spraying the entire epidemic prone area is time consuming, expensive, and it is difficult to cover all mosquito habitats. And also, it is discouraged on account of environmental pollution. While the biting activity of JE mosquitoes is at dusk, use of bed nets at night is likely to be ineffective and it is not the best solution for JE control. Segregating, slaughtering, or vaccinating pigs is economically not feasible and also difficult in other logistic angles. Other animals, like birds also act as amplifying hosts so even if pigs are eliminated JE will not disappear.

It seems that there is compelling evidence that human immunization is effective for containing the JE epidemics. Since the epidemic in man appears to follow epizootics and this coincides when the vector density shoots up in the anticipated epidemic districts, feasibility of JE vaccination has to be considered as a preventive measure. In such situations, identifying the risk area and determining the target popula-

Table1. Mean Values of Climatic Variables and Altitude in JE Endemic and Epidemic Areas

\begin{tabular}{|c|c|c|c|c|}
\hline & \multicolumn{2}{|c|}{ Endemic districts $(\mathrm{n}=\mathbf{8 0})$} & \multicolumn{2}{|c|}{ Epidemic districts $(\mathbf{n}=95)$} \\
\hline & Mean & S.D. & Mean & S.D. \\
\hline \multicolumn{5}{|l|}{ Climatic variables } \\
\hline Temperature $\left({ }^{0} \mathrm{C}\right)$ & 25.46 & 2.45 & 25.95 & 2.10 \\
\hline Relative Humidity (\%) & 63.82 & 6.46 & 66.00 & 9.44 \\
\hline Rainfall (mm) & 1057.52 & 1207.21 & 1306.16 & 1176.36 \\
\hline Altitude (mtr) & 436.86 & 2211.09 & 289.66 & 613.31 \\
\hline
\end{tabular}


tion to be immunized are crucial. Since the JE vaccine is already available / produced in India, a routine JE immunization may be initiated in all areas identified for the risk of epidemics.

Similarly, in the endemic districts the following control/preventive measures are suggested:

1. As the vector mosquito breeding places include mainly rice fields, the intermittent irrigation may be encouraged wherever possible, so that the fields are flooded only for a limited period.

2. Promoting the use of bio-control agents (fishes / 'Azolla', etc) and organic fertilizers ('neem' cakes) to reduce the mosquito immatures.

3. The likelihood of any sharp increase in vector abundance should be recognized in advance and resorted to appropriate preventive operations.

4. Monitoring viral activity in vector mosquitoes and reservoir animals is necessary to supplement with the information on vector abundance to predict the risk of transmission.

5. Rational use of appropriate insecticide in resting places of mosquitoes, viz; domestic and peri-domestic areas, cattle sheds, pigsties, chicken coops etc. to suppress the vector density in risk areas prior to the transmission.

6. Encouraging the use of suitable mosquito repellant(s), as a personal protection measure to reduce the man vector contact.

7. Since the children ( $<15$ years) alone are vulnerable in 'endemic' areas, they may be selectively considered for covering under the JE immunization programme.

However, each method has its own limitation, and it may not be prudent to depend on any single method. Therefore, an integrated management will be an ideal approach for JE prevention /control.

\section{CONCLUSIONS}

Review of data at macro-level showed that the occurrence of endemic JE is largely determined by the factors such as irrigation, agriculture activities, and animal husbandry practices; whereas the epidemic JE is mainly due to vagaries of climate conditions in the region. In the epidemic districts, both rainfall and altitude have a bearing on JE outbreak and thereby warrant preparedness to implement an effective preventive strategy such as JE immunization of target population in the risk area. In the endemic districts, an integrated approach in vector control has been suggested to reduce the transmission, besides selective immunization of children. Macro-level monitoring of environmental determinants and forecasting the risk of transmission could well be achieved, using the RS and GIS.

\section{ACKNOWLEDGEMENTS}

The authors greatly acknowledge Dr. M. Kalyanasundaram, Officer-in Charge, Vector Control Research Centre, Puducherry for providing necessary facilities. Thanks are also due to Dr. P. K. Das, Former Director, VCRC for his encouragement in undertaking this study.

\section{REFERENCES}

[1] Igarashi A. Epidemiology and control of Japanese encephalitis. World Health Statist Q (WHO) 1992; 45: 299-05.

[2] Umenai TR, Krzysko TA, Bektimirov, Assaad FA. Japanese encephalitis: Current Worldwide Status. Bull WHO 1985; 63: 625-31.

[3] WHO Japanese encephalitis (JE). Immunization service delivery and accelerated disease control. Programmes and projects. http://www.who.int/immunization_delivery/new_vaccines/je/en/ 20 April, 2008.

[4] Soman RS, Mourya DT, Mishra AC. Transovarial transmission of Japanese encephalitis virus in Culex vishnui mosquitoes. Ind J Med Res 1986; 84: 283-85.

[5] Sabesan S, Ravi R, Das PK. Elimination of lymphatic filariasis in India. Lancet Infect Dis 2005; 5: 4-5.

[6] Rajagopalan PK, Work TH. An Analysis of mosquito collections with special reference to the incidence and prevalence of 'Culex Vishnui Complex' in the Japanese encephalitis infected localities of North Arcot District, Madras State, India, from December 1955 through December 1957. Indian J Med Res 1969; 57: 1409-19.

[7] Lindsay SW, Wilkins HA, Zieler HA, Daly RJ, Petrarca V, Byass P. Ability of Anopheles Gambiae mosquitoes to transmit malaria during the dry and wet seasons in an area of irrigated rice cultivation in the gambia. J Trop Med Hyg 1991; 94: 313-24.

[8] Beck LR, Rodriguez MH, Dister SW, et al. Remote sensing as a landscape epidemiologic tool to identify villages at high risk for malaria transmission. Am J Trop Med Hyg 1994; 51: 271-80.

[9] Rosen L, Tesh RB, Lien JC, Cross JH. Transovarial transmission of Japanese encephalitis virus by mosquitoes. Science 1978; 199: 909-11.

[10] National Vector Borne Disease Control Programme. Japanese encephalitis. Ministry of Health \& Family welfare, Govt. of India. http://www.nvbdcp.gov.in/je.html. 20 April, 2008.

[11] Rajagopalan PK, Panicker KN. A Note on the 1976 Epidemic of Japanese encephalitis in Burdwan District, West Bengal. Indian J Med Res 1978; 68: 393-98.

[12] Hay SI, Tucker CJ, Rogers DJ, Packer MJ. Remotely sensed surrogates of meteorological data for the study of the distribution and abundance of arthropod vectors of disease. Ann Trop Med Parasitol 1996; 90: 1-19.

[13] Malone JB, Bergquist NR, Huh OK, et al. A global network for the control of snail-borne disease using satellite surveillance and geographic information systems. Acta Tropica 2001; 79: 7-12.

[14] Bergquist NR. Vector-borne parasitic diseases: new trends in data collection and risk assessment. Acta Tropica 2001; 79: 13-20. 